\title{
Calculations of the Refractive Index of AIGaN/GaN Quantum Well
}

\author{
Aleksandra B. Djurišić, Y. Chan, and E. Herbert Li \\ ${ }^{a}$ Department of Electrical \& Electronic Engineering, University of Hong Kong, \\ Pokfulam Road, Hong Kong
}

\begin{abstract}
We have calculated the refractive index of a $\mathrm{Al}_{\mathrm{x}} \mathrm{Ga}_{1-\mathrm{x}} \mathrm{N} / \mathrm{GaN}$ square quantum well $(\mathrm{QW})$. The imaginary part of the dielectric function has been obtained by summing up the contributions of the dominant interband transitions, excitonic contributions, and the continuum contribution, obtained by weighting the well's and the barrier's continuum contributions. In the calculation of the contribution of the conduction-valence band bound-state effect without electron-hole interaction, conduction bands are assumed to be parabolic and valence bands have been calculated using Chuang's model [Phys. Rev. B 54, 2491 (1996)], but with Chan's basis expansion method [J. Phys. C 19, L125 (1986)] instead of finite-difference scheme. Excitonic contribution has been described with an expression derived by the density-matrix approach at the subband edge without the influence of band mixing. The continuum contributions have been described with the modified Adachi's model. The effects of the aluminum mole fraction $x$ and the width of the well on the refractive index are analyzed and discussed.
\end{abstract}

Keywords: Refractive index, $\mathrm{Al}_{\mathrm{x}} \mathrm{Ga}_{1-\mathrm{x}} \mathrm{N} / \mathrm{GaN}$ quantum wells

\section{INTRODUCTION}

$\mathrm{Al}_{\mathrm{x}} \mathrm{Ga}_{1-\mathrm{x}} \mathrm{N}$ alloys have recently attracted a lot of interest for the development of optoelectronic devices operating in the visible and ultraviolet region, ${ }^{1,2}$ since the band gap of an alloy can vary from $3.45 \mathrm{eV}(x=0)$ to $6.28 \mathrm{eV}(x=1)$. Furthermore, the group-III nitrides are characterized by high-ionicity, very short bond lengths, low compressibility and high thermal conductivity. Such physical and structural properties are unique among III-V compounds. Since a precise understanding of the optical properties of these materials is essential to effective device operation and design, it is necessary to develop a comprehensive model which deals with the theoretical calculation of the refractive index. In this work, the effects of mole fraction (x) of aluminum and the width of the $\mathrm{GaN}$ well layer on the refractive index of $\mathrm{Al}_{\mathrm{x}} \mathrm{Ga}_{1-\mathrm{x}} \mathrm{N} / \mathrm{GaN}$ square quantum well (QW) are analyzed. To calculate the refractive index, both the real and imaginary parts of the dielectric function $\varepsilon(E)$ as a function of the photon energy $E=\hbar \omega$ over a wide spectral range are used. All transitions, including the excitonic effect, are taken into consideration in describing the QW system in a spectral range between $1 \mathrm{eV}$ and $9 \mathrm{eV}$.

Based on the band structure of $\mathrm{Al}_{\mathrm{x}} \mathrm{Ga}_{1-\mathrm{x}} \mathrm{N} / \mathrm{GaN}$ calculated via the $\mathrm{k} \cdot \mathrm{p}$ method for wurtzite semiconductors, ${ }^{3}$ only dominant interband transitions are considered. The lowest direct band gap is denoted as $E_{0}$, and the other transitions at energy regions higher than that of $E_{0}$ are denoted as $E_{1 \beta}$ where $\beta=A, B$ and $C$. A full QW calculation is employed for the $E_{0}$ region where the QW effect is the most significant. A continuum state calculation is performed for the higher lying direct transitions in the energy region $E_{1 \beta}$ using the composite interband transition model. ${ }^{4}$ The contributions to the imaginary part of dielectric function $\varepsilon_{2}(E)$ from each of these dominant transitions are considered separately. Various alloy parameters required for calculation have been estimated by the interpolation between $\alpha-\mathrm{GaN}$ and AlN materials. After summing up the contributions to the imaginary part, the real part of dielectric function $\varepsilon_{1}(E)$ is obtained by Kramers-Kronig transformation $(\mathrm{KKT})$. When the real and imaginary parts of the dielectric function are known, calculation of the refractive index is straightforward. The dependence of the calculated refraction index on the aluminum content $x$ and the width of the well layer in $\mathrm{Al}_{\mathrm{x}} \mathrm{Ga}_{1-\mathrm{x}} \mathrm{N} / \mathrm{GaN}$ square $\mathrm{QW}$ have been investigated.

This paper is organized as follows: the composite model is briefly described in section 2 . where each energy transition is considered separately. In section III, the estimation of parameters is described and results of the calculated refractive index are presented. Finally, a conclusion is drawn.

•E_mail: ehli@eee.hku.hk; Fax: +852 2559 8738; Tel: +852 28597091 


\section{DESCRIPTION OF THE MODEL}

The dielectric function depends on the electronic energy-band structure of the semiconductor QW material. Summing up all the contributions, $\varepsilon_{2}(\mathrm{E})$ is:

$$
\varepsilon_{2}(\omega)=\varepsilon_{2}^{E_{0}}(\omega)+\varepsilon_{2}^{E_{1 \beta}}(\omega)
$$

where $\varepsilon_{2}^{E_{0}}$ denotes the contribution to dielectric function from the low-lying direct gaps $\mathrm{E}_{0}$ and $\varepsilon_{2}^{E_{1 \beta}}$ denotes the contribution to dielectric function from $\mathrm{E}_{1 \beta}$ regions. This energy region corresponds to the band-edge region of the $\mathrm{QW}$. Our calculation includes the exciton and the bound-state effects, which are superimposed to the continuum contribution above the barrier gap energy. $\varepsilon_{2}^{E_{0}}$ is obtained by summing up all the above contributions, and it can be expressed as follows:

$$
\varepsilon_{2}^{E_{0}}(\omega)=\varepsilon_{2}^{\text {exc }}(\omega)+\varepsilon_{2}^{\text {bound }}(\omega)+\varepsilon_{2}^{E_{0} \text { cont }}(\omega)
$$

where $\omega$ is the frequency, $\varepsilon_{2}^{e x c}$ is the imaginary part of the dielectric function contributed by the QW 1S-exciton derived by the density-matrix approach at the subband edge, $\varepsilon_{2}^{\text {bound }}$ is the imaginary part of the dielectric function contributed by the conduction-valence band QW bound-states, and $\varepsilon_{2}^{E_{0} c o n t}$ is the imaginary part of the dielectric function determined by weighting the $\mathrm{AlGaN}$ well and GaN barrier continuum's at energies above the barrier energy in the MQW structure without the QW effect.

The QW subband edge states in the $\mathrm{E}_{0}$ region can be calculated separately for electrons and holes by an envelope function approximation ${ }^{5}$ using the Ben-Denial and Duke model. ${ }^{6}$ The imaginary part of the dielectric function contributed by the exciton effect, $\varepsilon_{2}^{e x c}(\omega)$, is based on the $1 \mathrm{~S}$ bound exciton wave function determined by a perturbative-variational method, ${ }^{7,8}$ using the separate electron and hole bound state at $\mathrm{k}_{\|}=0$ as the trial functions.

The imaginary part of the dielectric function contributed by the conduction-valence band bound-state effect without the electron-hole interaction, $\varepsilon_{2}^{\text {bound }}(\omega)$, is based on the direct interband transition around the absorption edge in the $\mathrm{E}_{0}$ region. $\varepsilon_{2}^{\text {bound }}(\omega)$ is expressed as:

$$
\varepsilon_{2}^{\text {bound }}(\omega)=\frac{e^{2}}{\varepsilon_{0} m_{0}^{2} \omega^{2} L_{z}} \sum_{p, q} \int\left|\hat{e} \cdot M_{p q}(k)\right|^{2} d k \frac{1}{4 \pi^{2}} \times \mathrm{L}\left[E_{p}(k)-E_{q}(k)-\hbar \omega\right],
$$

where $\mathrm{M}_{\mathrm{pq}}$ is the interband momentum-matrix element for the wurtzite materials, ${ }^{9} \mathrm{k}$ is the transverse wave vector in the direction parallel to the QW layer; $\mathrm{L}_{\mathrm{z}}$ is the well width of the QW and $\mathrm{L}$ is the Lorentzian broadening factor with halfwidth-half-maximum $\Gamma_{b}$.

The conduction bands are assumed parabolic; for the valence band calculation, we adopt Chuang's model. ${ }^{3}$ However, instead of using the finite-difference scheme stated there, we use Chan's basis expansion method. ${ }^{10} \mathrm{~A}$ total of 30 band-edge wavefunctions at $k_{t}=0$ are determined for the expansion in the calculation of the wavefunction at $k_{t} \neq 0$. The momentum matrix element in (3) for $\mathrm{TE}$ ( $\hat{e}=\hat{x}$ or $\hat{y} \perp \mathrm{c}$ axis) and $\mathrm{TM}(\hat{e}=\hat{z}$ or $\hat{y} \| \mathrm{c}$ axis) polarisation can be simplified to:

$$
\hat{e} \cdot M_{p q}(k)=\left\langle\Psi_{p}^{v}\left|\hat{p}_{x}\right| \Psi_{q}^{c}\right\rangle
$$

and

$$
\hat{e} \cdot M_{p q}(k)=\left\langle\Psi_{p}^{v}\left|\hat{p}_{z}\right| \Psi_{q}^{c}\right\rangle
$$

respectively. For the detailed expression, please refer to Chuang's work. ${ }^{9}$ 
Derived by the density-matrix approach at the subband edge without the influence of band mixing, $\varepsilon_{2}^{e x c}(\omega)$ is expressed as:

$$
\varepsilon_{2}^{e x c}(\omega)=\frac{e^{2}}{\varepsilon_{0} m_{0}^{2} \omega^{2} L_{z}}\left|\hat{e} \cdot M_{11}(k)\right|^{2} \times\left|\psi_{1 s}(k=0)\right|^{2} \mathrm{~L}\left(E_{e x c}-\hbar \omega\right),
$$

where $E_{e x c}$ is the exciton transition energy.

The last step in calculating the dielectric function in the $\mathrm{E}_{0}$ region is to combine the above contributions with the continuum state contribution. ${ }^{4}$ The lowest direct gap region of the wurtzite type material is assumed to be a critical point of the threedimensional (3D) $\mathrm{M}_{0}$ type. Together with the assumption that the valence and conduction bands are parabolic, the contribution of this low energy gap to the dielectric function, for a single layer (barrier or well), is expressed as:

$$
\varepsilon^{E_{0} c o n t}(\omega)=A E_{0}^{-3 / 2} \chi_{0}^{-2}\left[2-\left(1+\chi_{0}\right)^{1 / 2}-\left(1-\chi_{0}\right)^{1 / 2}\right]+\varepsilon^{e x-c o n t}(\omega)
$$

where

$$
\chi_{0}=\frac{E+i \Gamma_{0}}{E_{0}} .
$$

$A$ and $\Gamma_{0}$ are the strength and damping constants of the $E_{0}$ transition, respectively. Excitonic contributions at $E_{0}$ critical point are given by

$$
\varepsilon^{e x-c o n t}(\omega)=\sum_{m=1}^{\infty} \frac{A_{0}^{e x}}{m^{3}} \frac{1}{E_{0}-\left(G_{0}^{3 D} / m^{2}\right)-E-i \Gamma_{0}},
$$

where $A_{0}^{e x}$ is the three-dimensional (3D) exciton strength parameter, and $G_{0}^{3 D}$ is the $3 \mathrm{D}$ exciton binding energy. The $E_{0}$ critical point is actually composed of three $E_{0 A}, E_{0 B}$, and $E_{0 C}$ critical points. However, the splitting energies are very small $\left(E_{B A}=5.11 \mathrm{meV}, E_{C B}=18.77 \mathrm{meV}\right),{ }^{11}$ so that those three critical points can be treated as a single degenerate one. Extended absorption tails are inherent to all the models using Lorentzian broadening function due to its large wings. It was shown that by replacing the damping constant $\Gamma_{j}$ with the frequency dependent one, better agreement with experimental data can be achieved for both models of Kim et al. ${ }^{12}$ and of Rakić and Majewski ${ }^{13}$ for the zincblende semiconductors. Therefore, in the model for hexagonal semiconductors employed here we replace damping constants $\Gamma_{j}(j=0,1 A, 1 B, 1 C)$ in Eqs. (1)(6) with the expression

$$
\Gamma_{j}^{\prime}(E)=\Gamma_{j} \exp \left[-\alpha_{j}\left(\frac{\left(E-E_{j}\right)}{\Gamma_{j}}\right)^{2}\right]
$$

The concept of the frequency dependent damping enables us to vary the broadening function over a range of functions with similar kernels and different wings by changing the $\alpha_{\mathrm{j}} / \Gamma_{j}$ ratio. In such manner, overestimation of the absorption in the vicinity of a critical point can be avoided, without any initial assumption about the broadening type for the transitions at that critical point.

In the calculation of the quantum well, the $\varepsilon^{E_{0} \text { cont }}(\omega)$ is calculated by weighting the barrier and the well by a ratio of the well width $\left(\mathrm{L}_{\mathrm{z}}\right)$ to the barrier width $\left(\mathrm{L}_{\mathrm{b}}\right)$ :

$$
\varepsilon^{E_{0} \text { cont }}(\omega)=\frac{1}{L_{z}+L_{b}}\left[L_{z} \varepsilon_{(\text {well })}^{E_{0} \text { cont }}(\omega)+L_{b} \varepsilon_{(\text {barrier })}^{E_{0} \text { cont }}(\omega)\right] .
$$

Here, $\mathrm{L}_{\mathrm{b}}$ is taken to be $100 \AA$ for a MQW structure. Thus, contribution of the continuum state transitions to the imaginary part $\varepsilon_{2}^{E_{0} c o n t}$ of the dielectric function is given by taking the imaginary part of Eq. (11):

$$
\varepsilon_{2}^{E_{0} \text { cont }}(\omega)=\operatorname{Im}\left(\varepsilon^{E_{0} \text { cont }}\right)
$$


The energy transitions in higher energy regions are denoted as $E_{1 \beta}$. With the assumption that the quantum well effect can be neglected here, only the continuum state contribution is considered in the calculation of the dielectric function. Contributions of the critical points $E_{1 \beta}$ are given by: ${ }^{11}$

$$
\varepsilon^{E_{1} c o n t}(\omega)=-\sum_{\beta=A, B, C} B_{1 \beta} \chi_{1 \beta}^{-2} \ln \left(1-\chi_{1 \beta}^{2}\right)+\varepsilon_{1 X}(E)
$$

where

$$
\chi_{1 \beta}=\frac{E+i \Gamma_{1 \beta}}{E_{1 \beta}},
$$

while $B_{1 \beta}$ and $\Gamma_{1 \beta}$ are the strength and the damping constants of the $E_{1 \beta}$ transitions, respectively. Contributions of the Wannier-type two-dimensional (2D) excitons (discrete series of exciton lines at the $E_{1 \beta}$ critical points) are given by: ${ }^{11}$

$$
\varepsilon_{1 X}(E)=\sum_{\beta=A, B, C} \sum_{m=1}^{\infty} \frac{B_{1 \beta}^{X}}{(2 m-1)^{3}} \frac{1}{E_{1 \beta}-\left(G_{1 \beta}^{2 D} /(2 m-1)^{2}\right)-E-i \Gamma_{1 \beta}},
$$

where $B_{1 \beta}^{X}$ and $G_{1 \beta}^{2 D}$ are the strengths and binding energies of the excitons at $E_{1 \beta}$, respectively. The final $\varepsilon^{E_{1} \text { cont }}(\omega)$ is obtained in the same way as continuum contribution of the $\mathrm{E}_{0}$ transition, that is, by weighting the barrier and well regions by a ratio of well width $\left(\mathrm{L}_{\mathrm{z}}\right)$ to barrier width $\left(\mathrm{L}_{\mathrm{b}}\right)$. Thus, the imaginary part $\varepsilon_{2}^{E_{1} \text { cont }}$ of the continuum contribution to the dielectric function in the $\mathrm{E}_{1 \beta}$ region is given by:

$$
\varepsilon_{2}^{E_{1} \text { cont }}(\omega)=\operatorname{Im}\left(\varepsilon^{E_{1} c o n t}\right)
$$

The imaginary part of the total dielectric function $\varepsilon_{2}(\omega)$ is obtained by summing up the contributions from $\mathrm{E}_{0}$ and $\mathrm{E}_{1 \beta}$ regions given by Eqs. (3), (6), (12), and (16). The real part of the dielectric function is obtained by performing KKT on $\varepsilon_{2}(\omega)$.

When the dielectric function is known, real and imaginary parts of the index of refraction are readily calculated from the formulae:

$$
n(E)=\left[0.5\left(\varepsilon_{1}(E)+\left(\varepsilon_{1}^{2}(E)+\varepsilon_{2}^{2}(E)\right)\right)^{1 / 2}\right]^{1 / 2},
$$

and

$$
k(E)=\left[0.5\left(-\varepsilon_{1}(E)+\left(\varepsilon_{1}^{2}(E)+\varepsilon_{2}^{2}(E)\right)\right)^{1 / 2}\right]^{1 / 2}
$$

\section{RESULTS AND DISCUSSION}

In the bound state, exciton and continuum state calculations, a set of material parameter values is required. As for the bound state and exciton calculations, this includes parameters such as Luttinger-like valence-band effective mass parameters $A_{i}$ 's conduction-band effective mass for electrons and static dielectric constants. In the case when there are no data available for the ternary $\mathrm{Al}_{\mathrm{x}} \mathrm{Ga}_{1-\mathrm{x}} \mathrm{N}$ material parameters, the linear interpolation scheme, i.e. $\mathrm{P}\left(\mathrm{Al}_{\mathrm{x}} \mathrm{Ga}_{1-\mathrm{x}} \mathrm{N}\right)=\mathrm{xP}(\mathrm{AlN})+(1-\mathrm{x}) \mathrm{P}(\mathrm{GaN})$, is used. For the continuum state calculations, we have used the experimental data of Whetkamp et al. ${ }^{14}$ which have been modeled as a function of energy and composition. ${ }^{15}$ Whetkamp et al. ${ }^{14}$ determined the dielectric function of MOCVD grown $\mathrm{Al}_{\mathrm{x}} \mathrm{Ga}_{1-\mathrm{x}} \mathrm{N}$ using spectroscopic ellipsometry. The data have not been corrected for the surface roughness. Figs. 1 and 2 show the energy dependence of the real and imaginary parts of the index of refraction of $\mathrm{Al}_{\mathrm{x}} \mathrm{Ga}_{1-\mathrm{x}} \mathrm{N}$ alloy for different compositions, respectively. Excellent agreement between the model employed and the experimental data of Whetkamp et $a l .,{ }^{14}$ can be clearly observed for all compositions. 


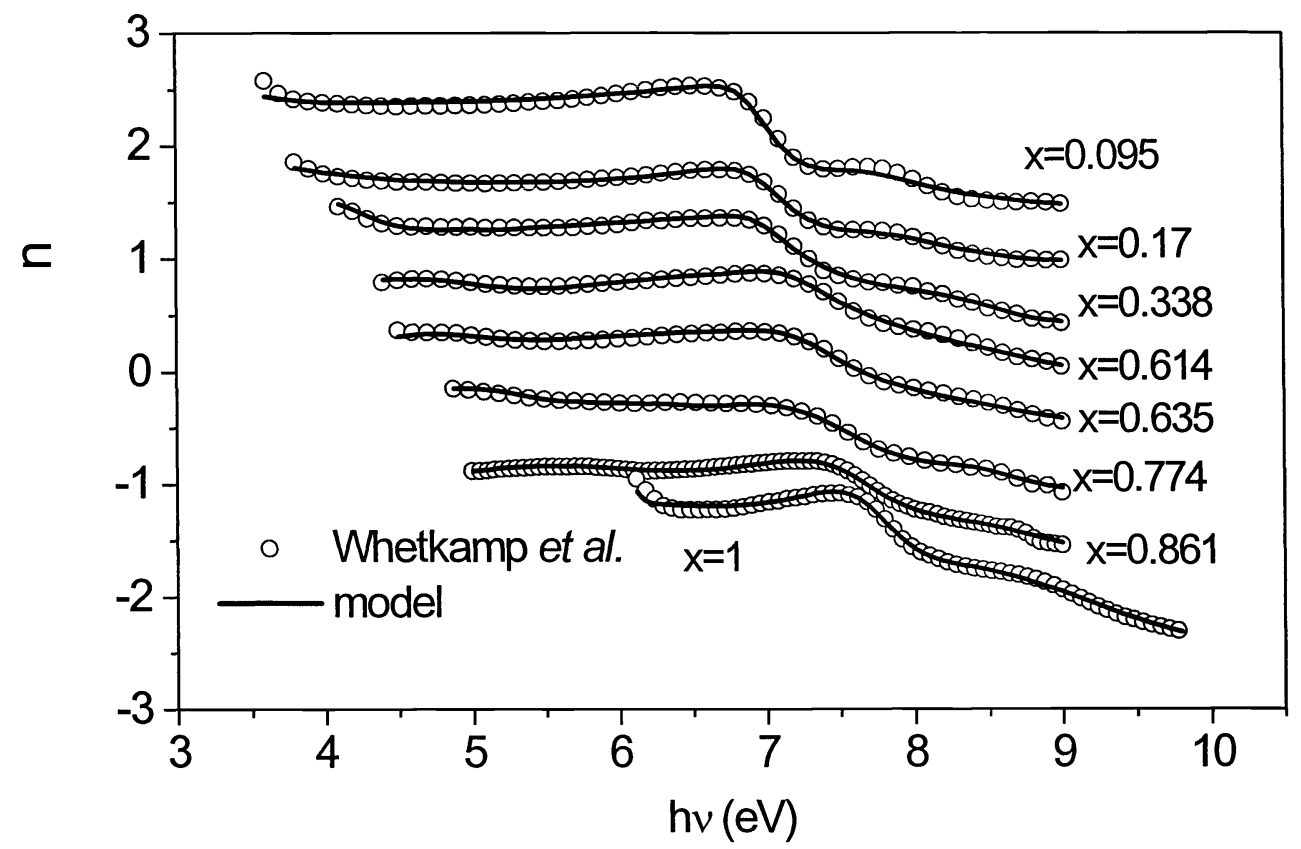

Fig. 1 The real part of the index of refraction as a function of energy for compositions $x=0.095,0.170,0.338,0.405,0.614,0.635,0.774$, 0.861 , and 1 . Circles - experimental data, ${ }^{14}$ solid line - model. ${ }^{15}$

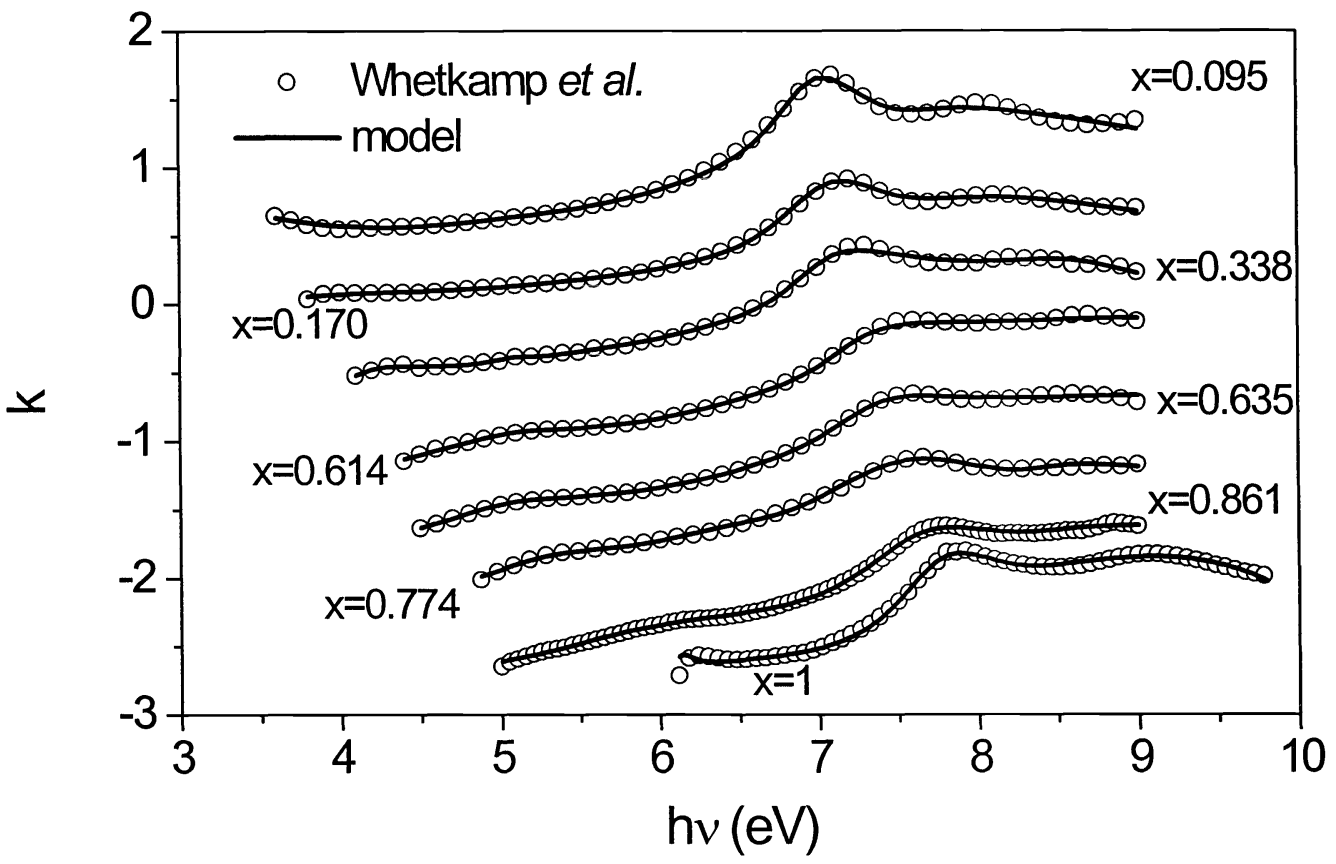

Fig. 2.The imaginary part of the index of refraction as a function of energy for compositions $x=0.095,0.170,0.338,0.405,0.614,0.635$, $0.774,0.861$, and 1. Circles - experimental data, ${ }^{14}$ solid line - model. ${ }^{15}$ 


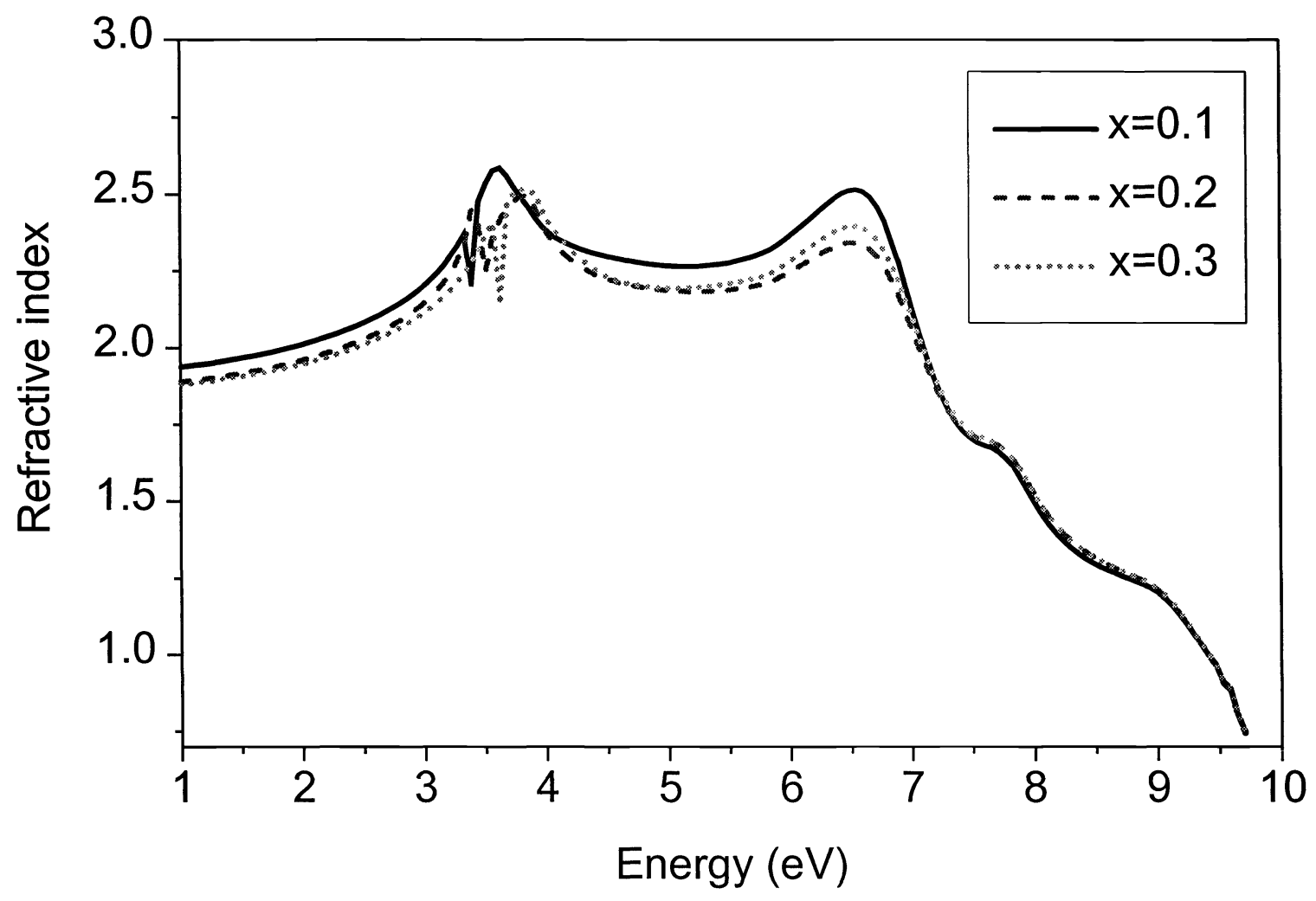

Fig. 3 TE polarization refractive index spectra of $\mathrm{Al}_{x} \mathrm{Ga}_{1-\mathrm{x}} \mathrm{N} / \mathrm{GaN} \mathrm{MQW}$ with $\mathrm{L}_{z}=60 \AA$ for $\mathrm{x}=0.1,0.2$ and 0.3 .

Parameters describing the dielectric function of the continuum contribution of $\mathrm{Al}_{x} \mathrm{Ga}_{1-\mathrm{x}} \mathrm{N}$ as a function of energy and aluminum content are given in Ref. ${ }^{15}$ Each parameter has been assumed to be cubic polynomial of Al content $x$. Bandgap dependence on aluminum content $x$ has been estimated in the same manner as in Ref., ${ }^{15}$ while effective mass parameters have been estimated according to data listed in Ref. ${ }^{16}$ The refractive index spectra of AlGaN/GaN MQW with well width equal to $60 \AA$ and $100 \AA$ and $A 1$ content equals $0.1,0.2,0.3$ are now readily determined at room temperature according to the model developed. The theoretical results of the room temperature refractive index for $\mathrm{Al}$ content $\mathrm{x}=0.1,0.2$ and 0.3 in the energy range from $1 \mathrm{eV}$ to $9 \mathrm{eV}$ with well width $\mathrm{L}_{z}=60 \AA$ and structures with well width $\mathrm{L}_{z}=100 \AA$ are presented in Fig.3 and Fig. 4 respectively.

\section{CONCLUSION}

In this work, the dependence of refractive index of the $\mathrm{Al}_{\mathrm{x}} \mathrm{Ga}_{1-\mathrm{x}} \mathrm{N} / \mathrm{GaN}$ MQW structure on well width and barrier composition has been studied. The theoretical model presented here employs semi-analytical, and semi-empirical calculation schemes for the refractive index over a wide spectral range from $1 \mathrm{eV}$ to $9 \mathrm{eV}$ and takes into account the continuum state contribution and the QW effect including excitons in the structure. Our results indicate the expected general trend of the refractive index, and the well-defined peaks obtained in the spectra show the quantum confinement at the band edge of the QW structure. The results we have obtained should be able to provide reasonable estimate of the refractive index of $\mathrm{Al}_{\mathrm{x}} \mathrm{Ga}_{1-\mathrm{x}} \mathrm{N} / \mathrm{GaN}$ quantum wells for the design of photonic devices using this material system. 


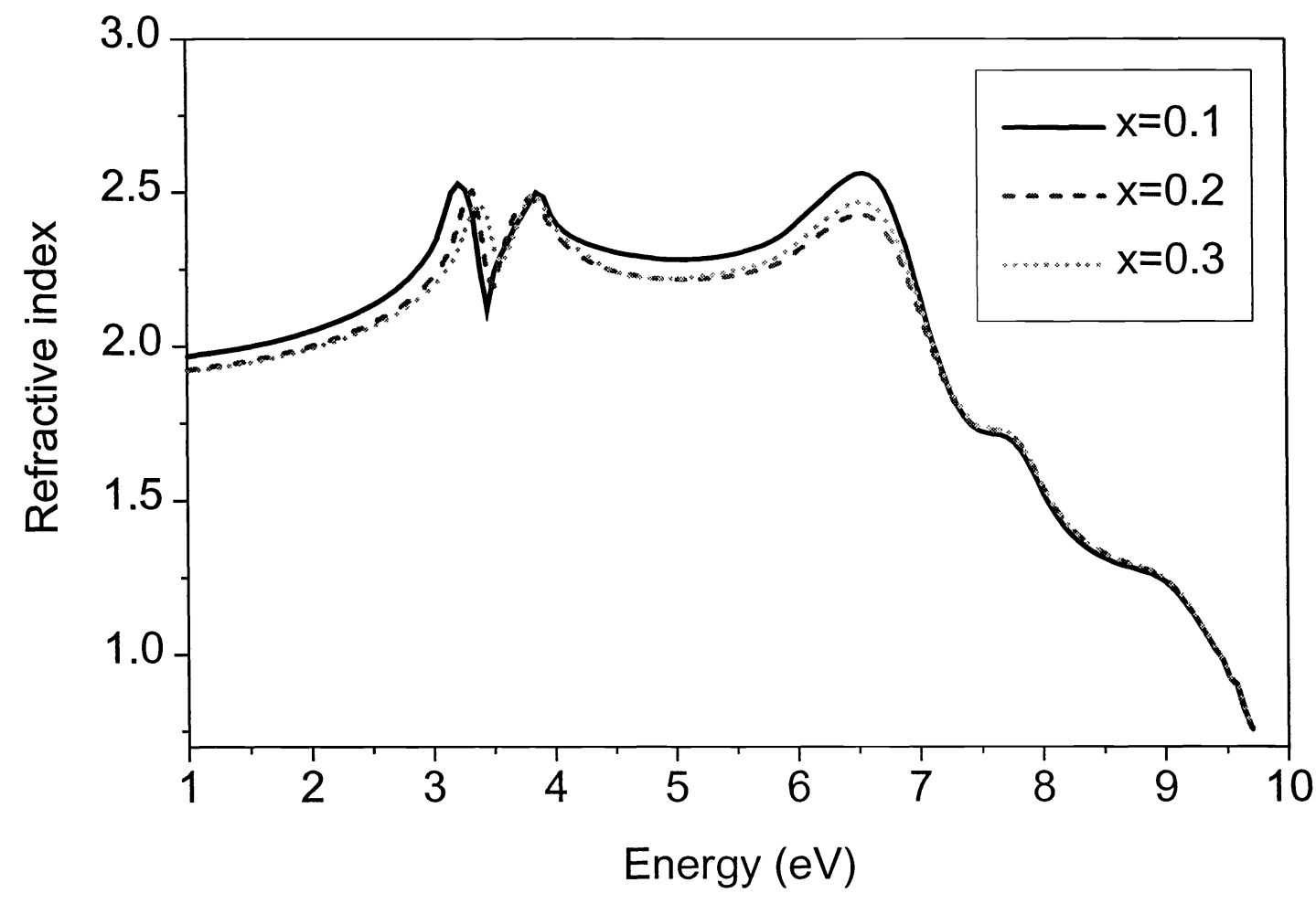

Fig. 4 TE polarization refractive index spectra of $\mathrm{Al}_{. x} \mathrm{Ga}_{1-\mathrm{x}} \mathrm{N} / \mathrm{GaN} \mathrm{MQW}$ with $\mathrm{L}_{\mathrm{z}}=100 \AA$ for $\mathrm{x}=0.1,0.2$ and 0.3 .

\section{ACKNOWLEDGEMENTS}

The authors would like to thank the RGC earmarked grant of Hong Kong and the University of Hong Kong CRCG research grant for financial support.

\section{REFERENCES}

1. I. Akasaki and H. Amano, "Widegap column-III nitride semiconductors for UV/blue light emitting devices", $J$. Electrochem. Soc., 141, pp. 2266-2271, 1994.

2. S. Nakamura and G. Fasol: The blue laser diode: GaN based light emitters and lasers (Springer, Berlin, 1997).

3. S. L. Chuang and C. S. Chang, Phys. Rev. B 54 (1996) 2491.

4. A. B. Djurišić and E. H. Li, "Modeling the optical constants of hexagonal GaN, InN and AlN", J. Appl. Phys., 85, pp. 2848-2853, 1999.

5. G. Bastard, J.A. Brum, and R. Ferreira, "Electronic states in semiconductor heterostructures" in Solid State PhysicsAdvances in Research and Applications, H.Ehrenreich and D. Tumbull, (Eds.) 44 (Academic Press, New York, 1991) p229-415.

6. D.J. Ben-Daniel and C.B. Duke, Phys. Rev. 152 (1966) 683.

7. Y.C. Lee, W.N. Mei and K.C. Lui, J. Phys. C 15 (1982) L469.

8. T.F.Jiang, Solid State Commun. 50 (1984) 589.

9. S.L. Chuang, IEEE J. Quantum Electron. 32 (1996) 1791.

10. K.S. Chan, J. Phys. C 19 (1986) L125. 
11. T. Kawashima, H. Yoshikawa, S. Adachi, S. Fuke and H. Ohtsuka, "Optical properties of hexagonal GaN", J. Appl. Phys., 82, pp. 3528-3535, 1997.

12. C. C. Kim, J. W. Garland, and P. M. Raccah, "Modeling the optical dielectric function of semiconductors: extension of the critical-point parabolic-band approximation", Phys. Rev. B, 45, pp. 11749-11767, 1992.

13. A. D. Rakić and M.L. Majewski, "Modeling the optical dielectric function of GaAs and AlAs: extension of Adachi's model", J. Appl. Phys., 80, pp. 5909-5914, 1996.

14. T. Whetkamp, K. Wilmers, N. Esser, W. Richter, O. Ambacher, H. Angerer, G. Jungk, R. L. Johnson and M. Cardona, "Spectroscopic ellipsometry measurements of $\mathrm{Al}_{\mathrm{x}} \mathrm{Ga}_{1-\mathrm{x}} \mathrm{N}$ in the energy range 3-25 eV", Thin Solid Films 313-314, pp. 745-750, 1998.

15. A. B. Djurišić and E. H. Li, " Modeling the Optical Constants of $\mathrm{Al}_{\mathrm{x}} \mathrm{Ga}_{1-\mathrm{x}} \mathrm{N}$ Alloys ", J. Phys. Soc. Japan, to be published.

16. T.-Y. Chung and K. J. Chang, "Exciton binding energies in GaN/AlGaN quantum well structures", Semicond. Sci. Technol. 13, pp. 876-81, 1998. 